\title{
The effects of repeated shifts in magnitude of food reward upon the barpress rate in the rat
}

\author{
MITRI E. SHANAB, JULIA DOMINO, and LINDA RALPH \\ California State University, Fresno, California 93740
}

\begin{abstract}
In a four-shift experiment, four independent groups of hungry rats received the following treatments: LLLLL, SSSSS, LSLSL, and SLSLS, with each letter denoting the magnitude of food reward (large or small) received in each of the five phases of the experiment. Contrast effects were not obtained in the second shift. However, significant positive and negative contrast effects were found in the third shift. The effects started to weaken in the fourth shift, as reflected by a much delayed, albeit significant PCE and a graphical but nonsignificant NCE. The use of multiple shifts to assess the effects of learning and motivation factors on contrast was urged.
\end{abstract}

When subjects are shifted from a large to a smaller reward in the runway, they typically run significantly slower than subjects receiving the same small reward all the time. This undershooting phenomenon, commonly referred to as the negative contrast effect (NCE), is obtained rather regularly (Cox, 1975). Until recently, the opposite effect, an overshooting or positive contrast effect (PCE), has not been as easy to obtain in the runway, especially when single upshifts are made. In other words, subjects shifted from a small to a larger reward only once do not normally run faster than control subjects receiving large reward all the time, unless appropriate controls for the ceiling effect associated with the running response are observed (Mellgren, 1972; Shanab \& Biller, 1972; Shanab, Sanders, \& Premack, 1969). More recently, the PCE has been reported in multiple-shift designs in which subjects are either shifted from large to small before being shifted back to large reward or they receive repeated shifts from small to large reward (Benefield, Oscos, \& Ehrenfreund, 1974; Shanab, France, \& Young, 1976; Shanab, Young, \& France, 1975). The preceding studies utilized the runway. There are only two studies that investigated the effects of repeated shifts in food reward upon the barpress response. Wookey (1976, Experiment 2) found a PCE based on the barpress only when his subjects were shifted from large to small reward during magazine training before receiving large reward for leverpressing. However, no PCE was obtained when subjects were shifted from small reward received during magazine training to large reward received during barpress training. A NCE was obtained both when subjects were shifted during magazine training from small to large reward before receiving small reward for leverpressing, as well as when subjects were shifted from large reward

Reprint requests should be sent to Mitri E. Shanab, Department of Psychology, California State University, Fresno, California 93740. during magazine training to small reward during barpress training. Unfortunately, Wookey did not include control subjects that received differential reward magnitudes for barpressing only. In a two-shift design in which only the barpress was differentially reinforced, Shanab, Kong, and Domino (1977) reported a strong NCE in the second shift, but only a graphical, although nonsignificant, PCE. The absence of a statistically significant PCE was interpreted in part as due to a possible ceiling effect induced by the discrete-trials FI 30 -sec schedule used in the study. The upshifted rats in the previous study (Shanab et al., 1977) respond at very high rates that were probably close to the subjects' physiological limit. Since a VI schedule generally produces low to moderate, but steady, rates of responding, it is less likely that subjects trained on such a schedule would respond close to their physiological limit, thus allowing for the emergence of any positive contrast effects. On the other hand, it is also possible that two shifts might not have been sufficient to produce the effect. In other words, the likelihood of the occurrence of a PCE is probably enhanced when subjects receive multiple shifts. This is based on the reasoning presented by Benefield et al., (1974), which was in turn based on Crespi's (1944) earlier theory of eagerness. According to these investigators, experience with frustration is a sufficient condition for PCE as well as NCE.

The present study was designed to account for these factors by using a VI schedule rather than a FI schedule and by giving the subjects four rather than two shifts. Thus, PCE would be obtained in subjects shifted to large reward only if these subjects had prior experience with a frustratingly small reward. Similarly, only if subjects had experienced small reward earlier in training would a shift from large to small reward produce frustration and thus lead to a NCE. In addition, subjects receiving four shifts would presumably experience frustration more frequently than those receiving only two shifts, hence they would be expected to exhibit both 
PCE and NCE more readily, especially under conditions that control for any ceiling effects.

\section{METHOD}

\section{Design}

The study consisted of four phases. In Phase 1, two groups of rats received either six $45-\mathrm{mg}$ pellets (large reward, $L$ ) or one 45-mg pellet (small reward, S) on a VI 30-sec schedule. In Phase 2, half of the subjects in each group were shifted to the other reward condition, while the remaining half continued to receive the same reward magnitude, thus yielding four groups: L-L, L-S, S-L, and S-S. In Phases 3, 4, and 5, subjects in Groups L-S and S-L received repeated shifts to their respective contrasting reward conditions, while Groups L-L and S-S remained unshifted. Thus, the final groups were: S-S-S-S-S, L-L-L-L-L, L-S-L-S-L, and S-L-S-L-S, with each letter denoting the reward magnitude received in the particular phase.

\section{Subjects}

The subjects consisted of 38 male albino rats of the SpragueDawley strain approximately 120 days old at the beginning of the experiment. Three rats died in Phase 4, one each from Groups L-L-L-L, L-S-L-S, and S-L-S-L. Another rat from the large-reward control group died in Phase 5.

\section{Apparatus}

Two identical Lehigh Valley Skinner boxes controlled by standard solid-state programming equipment were used. The boxes were modified to deliver either one or six $45-\mathrm{mg}$ Noyes food pellets. Each Skinner box was placed in a soundproof chamber equipped with an exhaust fan, which also served to mask external noises.

\section{Procedure}

Upon arrival from the supplier, the subjects were placed on free feeding and watering for $\mathbf{3 0}$ days and were handled for $3 \mathrm{~min} /$ day. Thereafter, and for the remainder of the experiment, the subjects were maintained on a $12-\mathrm{g} /$ day food allowance. After the rats had been on food deprivation for 2 weeks, they were randomly assigned to one of the two Skinner boxes and allowed to explore their box for $5 \mathrm{~min} /$ day for 2 days. As soon as magazine training was completed, each subject received five sessions of CRF training. Then the subjects were matched on the basis of their response rate over the last two sessions of CRF training and assigned to either a large- or small-reward condition. On Days 6 and 7, the subjects were tained on a FR 10- and VI $15-\mathrm{sec}$ reinforcement schedule, respectively, and received reward magnitudes appropriate for each of their designated conditions.

Phase 1 began on Day 8, at which time the subjects were reinforced on a VI 30-sec schedule. Each daily session consisted of six 30 -sec cycles, separated by 20 -sec time-out periods, during which the houselight and the cue lights above the food cup were off and the response counter was inoperative. The time-out periods began immediately after the reinforcement was deliverd. To control for familiarity with the schedule of reinforcement, four variations of a VI $30-\mathrm{sec}$ schedule were used and rotated daily.

Following stable performance in Phase 1, the subjects within each condition were matched on the basis of their overall response rate over the last three sessions of Phase 1 and divided in half, thereby yielding four reward conditions: S-S, L-L, S-L, and L-S. In Phases 3, 4, and 5, Phase 1, 2, and 3 conditions, respectively, were reinstated for the shifted subjects. Phases 1 and 5 consisted of 20 and 16 sessions, whereas Phases 2, 3, and 4 each lasted for 18 sessions. Phase 5 was reluctantly terminated before the subjects showed signs of stability, for the animal caretaker inadvertently placed all rats on free feeding following the 16 th session.

\section{RESULTS AND DISCUSSION}

All analyses are based on overall response rate during each 3-min session. The results of an analysis of variance test on mean response rate over the last four blocks in Phase 1 revealed no significant main effects $[\mathrm{F}(1,36)<1]$. A two-way analysis of variance was performed on the mean response rate over Blocks 3-9 in Phase 2, yielding significant reward magnitude main effect $[F(1,34)=11.49, p<.01]$. However, neither the shift main effect $(F<1)$ nor the Shift by Magnitude interaction effect were significant $[\mathrm{F}(1,34)=1.37$, $\mathrm{p}>.05]$. Individual comparisons showed neither a significant PCE $(F<1)$ nor a NCE $[F(1,34)=1.05$, $\mathrm{p}>.05$ ]. Unlike Phase 2, the design used in Phases 3-5 was no longer a pure factorial design. Therefore, the results of the main effects in each of these three phases are based on a one-way rather than a two-way analysis of variance. It should be noted that, since the study makes specific predictions, the relevant analysis could be based on simple effects only. However, both types of analyses are reported for these last three phases. Examination of the results in Phase 3 revealed no discernible differences. This was supported by the results of an analysis of variance over all blocks, which yielded a nonsignificant main effect $(F<1)$. A similar analysis over the last five blocks also yielded a nonsignificant main effect $[F(3,34)=1.28, p>.05]$. Neither a PCE nor a NCE were revealed by the results of a simple effects test on both sets of data.

As Figure 1 shows, the divergence of the shifted groups relative to the control groups in Phase 4 was quite clear and occurred rather early in the phase. The separation of the groups in Phase 5 occurred more gradually and was not as marked as that in Phase 4 . The results of an analysis of variance over Blocks 3-9 of Phase 4 yielded a significant main effect $[\mathrm{F}(3,33)=7.20$, $\mathrm{p}<.01$ ] . Individual comparisons revealed both a significant PCE $[F(1,33)=4.38, p<.05]$ and a significant NCE $[F(1,33)=4.19, p<.05]$. Separate analyses of variance followed by tests of simple effects were performed on Blocks 2-8, 4-8, 5-8, 6-8, and 7-8 of Phase 5, yielding nonsignificant contrast effects. Since all these analyses yielded highly similar results, only the results of the test over Blocks 5-8 will be reported. A significant main effect was found $[F(3,32)=6.09, p<.01]$. Individual comparisons showed neither a significant PCE nor NCE $[\operatorname{Fs}(1,32)=3.04$ and 1.45 , both ps $>.05$, respectively]. However, the results of an analysis of variance over the last three session (i.e., 1.5 blocks) yielded a significant main effect $[\mathrm{F}(3,32)=7.89$, $\mathrm{p}<.01]$. Individual comparisons showed a significant PCE $[F(1,32)=4.37, p<.05]$, but a nonsignificant $\operatorname{NCE}[F(1,32)=1.70, p>.05]$.

In summary, then, no contrast effects were obtained during the first shift, which is consistent with predictions. The lack of such effects in the second shift (Phase 3) was not expected. The fact that very clear and 


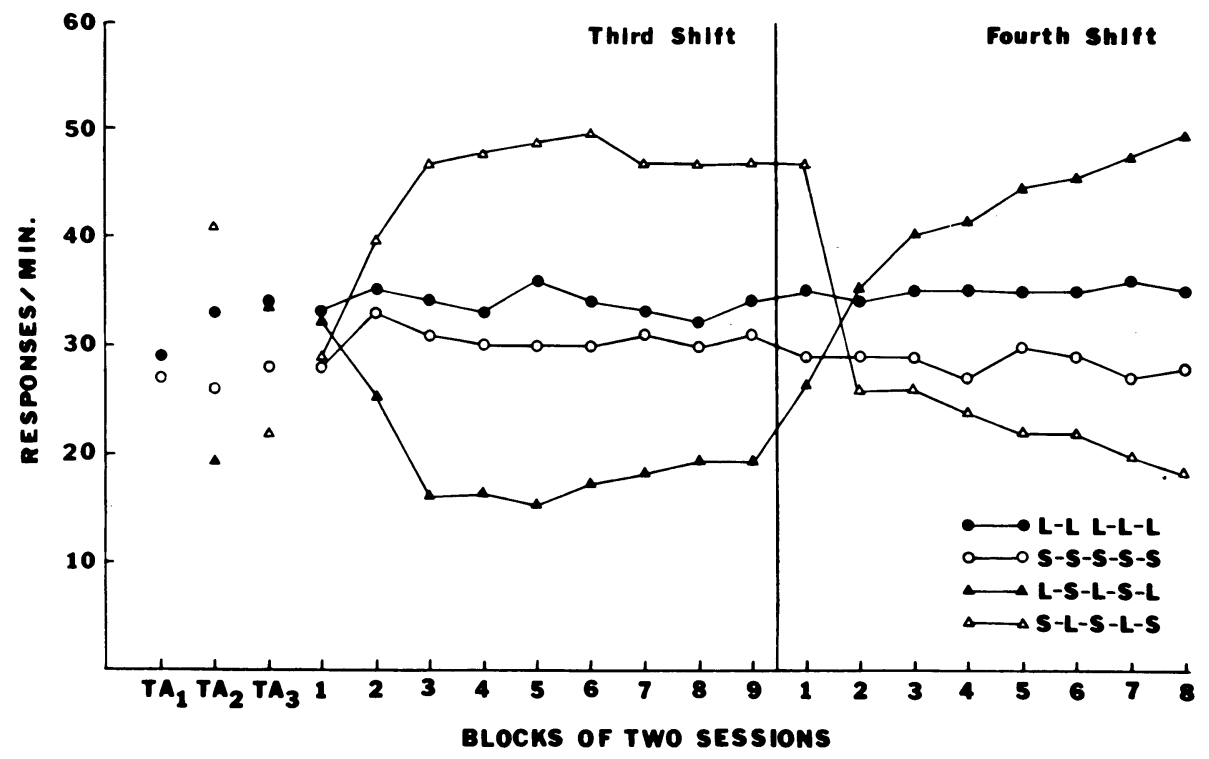

Figure 1.

sustained positive and negative contrast effects were observed in the third shift (Phase 4) suggests that the lack of significant differences during the second shift could reflect either a statistical artifact or the fact that not enough sessions were run in Phase 3. The latter explanation in particular could account for both the difficulty of obtaining significant contrast effects during most of Phase 5 and the late emergence of contrast in that phase. However, such an interpretation cannot account for the rather fast and sustained contrast effects observed during the third shift. It is conceivable that with several repeated shifts the subjects learn to discriminate the alternating pattern of shifting reward magnitudes. In frustration terms, this means that the subject would experience less frustration following several repeated shifts, thus an attenuation of contrast effects would be expected. The persistence of contrast during the third shift suggests that some form of learned frustration is probably involved. If these sustained contrast effects reflect a learning rather than a performance effect, then subjects shifted in Phase 5 would be expected to continue to respond for some time as they did in Phase 4 before learning to respond appropriately to the new reward conditions. This persistence would thus account for the slow and gradual emergence of contrast in Phase 5.

The finding of a PCE in this study supports the notion the the VI schedule can serve as a control procedure for the ceiling effects that are presumably present whenever high-response-rate-producing schedules are used. The FI schedule used in the earlier study (Shanab et al., 1977) yielded very high rates of responding, thus precluding the appearance of a significant PCE. The present findings of contrast effects with repeated shifts suggest that the effect of multiple shifts on contrast is not linear, but probably curvilinear. The latter in turn suggests that contrast is not a simple motivational phenomenon, but is probably complexly determined by both learning and motivational factors. It would seem that the use of the multiple-shifts paradigm in the future would greatly enhance our understanding of the role of these factors in contrast.

\section{REFERENCES}

Benefield, R., Oscos, A., \& Ehrenfreund, D. Role of frustration in successive positive contrast. Journal of Comparative and Physiological Psychology, 1974, 86, 648-651.

Cox, W. M. A review of recent incentive contrast studies involving discrete-trial procedures. The Psychological Record, 1975, 25, 373-393.

Crespi, L. P. Amount of reinforcement and level of performance. Psychological Review, 1944, 51, 305-309.

Mellaren, R. L. Positive and negative contrast effects using delayed reinforcement. Learning and Motivation, 1972, 3, 185-193.

Shanab, M. E., \& Biller, J. D. Positive contrast in the runway obtained following a shift in both delay and magnitude of reward. Learning and Motivation, 1972, 3, 179-184.

Shanab, M. E., France, J., \& Young, T. Positive and negative contrast effects obtained following shifts in liquid sucrose reward in thirsty rats. Animal Learning \& Behavior, 1976, 4, 9-12.

Shanab, M. E., Kong, J., \& Domino, J. Incentive contrast following repeated shifts in magnitude of food reward in the Skinner box. Bulletin of the Psychonomic Society, 1977, 9, 47-50.

Shanab, M. E., Sanders, R., \& Premack, D. Positive contrast in the runway obtained with delay of reward. Science, 1969, 164, 724-725.

Shanab, M. E., Young, T., \& France, J. Negative contrast as a function of downshifts in magnitude of sucrose concentrations in thirsty rats. Bulletin of the Psychonomic Society, 1975, 5, 381-384.

WookEY, P. E. Transfer and non-transfer successive incentive contrast effects in the rat. Quarterly Journal of Experimental Psychology, 1976, 28, 541-551.

(Received for publication March 6, 1978.) 